Meta

Journal des tradlucteurs

Translators' Journal

\title{
La lecture critique en traductologie
}

\section{Daniel Gile}

Volume 40, numéro 1, mars 1995

URI : https://id.erudit.org/iderudit/002894ar

DOI : https://doi.org/10.7202/002894ar

Aller au sommaire du numéro

Éditeur(s)

Les Presses de l'Université de Montréal

ISSN

0026-0452 (imprimé)

1492-1421 (numérique)

Découvrir la revue

Citer cet article

Gile, D. (1995). La lecture critique en traductologie. Meta, 40(1), 5-14.

https://doi.org/10.7202/002894ar d'utilisation que vous pouvez consulter en ligne.

https://apropos.erudit.org/fr/usagers/politique-dutilisation/ 


\section{LA LECTURE CRITIQUE EN TRADUCTOLOGIE}

DANIEL GiLE

INALCO el ISIT, Paris, france

\section{INTRODUCTION}

Dans sa marche vers un statut universitaire de discipline autonome, voire indépendante, la traductologie, qui a été pendant longtemps méditative et spéculative, fait de plus en plus souvent appel à des considérations et à des normes scientifiques telles qu'elles sont définies dans des disciplines de recherche plus anciennes. C'est ce que l'on voit notamment. en ce qui concerne la traduction écrite, à travers les récents travaux de l'école dite descriptive ( $c f$. Toury 1985: Hermans 1985), les efforts soutenus de la Finlandaise Sonja Tirkkonen-Condit (Tirkkonen-Condit 1991: Tirkkonen-Condit et Laffling 1993) en faveur de la recherche empirique, les travaux de Candace Séguinot (1989) et Jeanne Dancette (1990) au Canada, de H.-P. Krings (1988) et Wolfgang Lörscher (1991) en Allemagne, pour ne citer que les exemples les plus marquants. En interprétation aussi, un paradigme plus scientifique que par le passé se fait jour ( $c f$. Moser-Mercer 1991; Gran et Taylor 1990; Gile 1993).

À theure actuelle, les départements universitaires de traductologie dans le monde sont rares; la plupart des traductologues ne bénéficient pas d'une formation méthodo. logique à la recherche, et c'est en grande partie par mimétisme que les jeunes chercheurs acquièrent leur savoir-faire. D'où l'importance d'une certaine capacité de discrimination dans la lecture des articles et autres textes de recherche. Dans cet article, nous nous proposons d'énoncer quelques principes de lecture critique de ce type de textes. Nos références sont notre propre formation a la recherche (en mathématiques, puis en sociologie) et notre activité de chercheur, mais aussi les observations recueillies au fil de notre activité d'interprète à de nombreuses conférences scientifiques s "inscrivant dans différentes disciplines des sciences de la nature et de l'homme, et surtout l'étude de textes sur les principes et méthodes de la recherche dans ces disciplines. Au cours de cet effort de lecture et d'observation. il nous est apparu qu'en dépit des débats philosophiques et querelles de chapelle sur l'existence ou l'inexistence d'une démarche scientifique et sur les différences entre les sciences dures et les sciences molles, il existe bien un noyau dur de règles sur lesquelles les scientifiques semblent être tous d'accord, une «...logique fondamentale sous-jacente à toute activité de recherche" (Robert 1988: 39; voir aussi Nagel 1967:6). Ces principes, que l'on trouve réitérés dans les textes sur les méthodes de recherche en psychologie, en sociologie, dans les sciences de l'éducation, en médecine et en biologie nous semblent éminemment applicables à la traductologie. Nous nous proposons d'intégrer ceux qui sont pertinents dans le corps de ce texte, qui vise à aider les jeunes chercheurs à retirer le maximum des articles de recherche qu'ils lisent - et ce à travers la lecture critique.

\section{POURQUOI LA LECTURE CRITIQUE ?}

L'une des caractéristiques fondamentales de la science est sa dimension critique (du moins, sous sa forme idéalisée, qui ne correspond pas toujours à la réalité. comme le montre le philosophe des sciences Thomas Kuhn ; voir aussi Chauvin 1981). D'après Karl 
Popper, la science doit être une révolution permanente. Pour les spécialistes des méthodes de recherche, la critique doit être au cơur même de l'entreprise scientifique (FrankfortNachmias el Nachmias 1992: 20). La lecture critique des textes de recherche est donc une partie intégrante du processus de recherche. En dehors des cas où elle a une fonction essentiellement évaluative (lecture d'une thèse par les membres du jury, d'un article par les membres du comité de lecture d'une revue), elle a un rôle d'agent correcteur et moteur dans le mécanisme de la progression scientifique (voir. par exemple. Babbie 1992). Elle doit notamment aider l'auteur du texte lui-même à avancer en le faisant bénéficier de critiques qui lui permettront de corriger, de compléter, d'améliorer. C'est le cas des mémoires universitaires et thèses, des articles soumis à sélection par les pairs (peer review'). et. moins directement, des textes dont les thèses et résultats font l'objet de comptes rendus critiques.

La mise à jour des faiblesses et points forts d'un travail scientifique permet aussi au lecteur de trouver de nouvelles questions de recherche, de nouvelles méthodes d'investigation qui sont autant de directions d'exploration qui s'ouvrent à lui; la science est collective et cumulative, chaque chercheur ajoutant ses pierres à l'édifice construit par la communauté (Moravscik 1980); Robert 1988). La lecture critique ne doit donc pas être perçue comme agressive, mais comme analytique et constructive. En principe, les chercheurs sont d'ailleurs heureux de bénéficier de critiques fondées : en traductologie, elles sont une denrée souvent trop rare.

Au-delà de son aspect institutionnel, dans sa fonction plus fondamentale d'apprentissage, la lecture critique, qui implique une analyse attentive du texte lu, permet de mieux le comprendre et mieux le retenir qu une lecture plus superficielle. Pour le chercheur débutant, elle est un puissant outil didactique, car elle lui indique les démarches à suivre. ainsi que les écueils à éviter. Notre cours d'initiation aux méthodes de recherche dans les études japonaises à l'INALCO, dont les étudiants s'intéressent à des sujets très variés et a priori étrangers l'un à l'autre, puisqu'ils relèvent tout autant de la littérature que de l'économie, la linguistique, le commerce ou les sciences et techniques, est composé pour une grande pant d'exercices de lecture critique d'articles, mémoires de maîtrise et thèses de doctorat. L'expérience nous a montré que si certaines lacunes des textes lus passaient inaperçues des lecteurs, essentiellement en raison de leur méconnaissance du sujet traité. les principales faiblesses méthodologiques, ainsi d ailleurs que les caractéristiques de présentation des travaux, ont en général été bien saisies par les étudiants après une orientation méthodologique. S'agissant d'une discipline donnés. en l'occurrence la traductologie, chaque lecteur dispose en principe de la motivation et de connaissances de base qui doivent lui permettre de retirer de la lecture critique bien davantage.

On ne négligera pas enfin le rôle très important de la lecture critique appliquée par l'auteur à son propre texte: elle est l'un des meilleurs moyens dont dispose tout chercheur pour améliorer celui-ci avant de le publier ou de le soumettre à un jury.

\section{COMPRENDRE L.E TEXTE:}

Un travail de recherche ne se fait pas dans le vide. Il aborde une problématique particulière. el étudie une question ou des questions précises avec des objectifs précis. Pour pouvoir le juger, il faut pouvoir le situer par rapport à ces repères. Concrètement, on s'orientera à travers des interrogations: Quelle est ou quelles sont les questions que se pose l'auteur dans son travail de recherche, ou quels sont l'idée ou le phénomène qu'il cherche à explorer'?

Quel est ou quels sont les objectifs précis de son travail?

Parmi les problématiques générales qui se posent en traductologie. citons l'éternelle question de la fidélité, celle de la formation. celle du statut social du traducteur, celle des 
différences entre traduction et interprétation, entre traduction littéraire et traduction non littéraire, celles du rapport entre traduction et linguistique, celle de la recherche d'une théorie générale de la traduction, ainsi que les problèmes spécifiques se posant dans la traduction d'une langue donné à une autre langue, des questions techniques sur la traduction de différents genres littéraires, et des problèmes méthodologiques dans la recherche traductologique.

En général, chaque projet de recherche aborde un aspect particulier de la problématique et essaie de répondre à une question précise qui permet d'avancer dans la réflexion ou dans la connaissance des phénomènes pertinents, mais sans prétendre résoudre l'ensemble des problèmes qui se posent.

Ainsi, un chercheur peut se situer dans l'exploration du fonctionnement mental de l'interprétation, et essayer de construire un modèle théorique de celle-ci. Il peut aussi avoir une hypothèse sur ce fonctionnement mental de l'interprétation, et viser dans son projet à la tester. Un autre chercheur peut se placer dans la problématique de la formation à la traduction, et s'interroger sur l'utilité de l'introduction de cours de théorie dans la formation du traducteur; dans le cadre de son travail, il peut chercher des éléments de réponse à sa question en comparant par exemple la progression des étudiants dans un cursus avec cours théorique avec celle des étudiants dans un cursus qui $n$ 'en comporte pas. Un troisième chercheur peut se demander si traduire vers une langue maternelle donne de meilleurs résultats que traduire vers une langue non maternelle, et étudier la différence dans les traductions produites chez un ou deux traducteurs.

Il est très important de comprendre les questions que se pose l'auteur ainsi que les objectifs précis de son travail, car on jugera sa méthode et ses conclusions en fonction de ceux-ci. On peut ne pas être d'accord sur le choix de la question fondamentale ou sur les objectifs de la recherche, mais ces critiques doivent rester «en amont»; la réalisation de l'objectif que s'est fixé l'auteur doit être jugée en fonction de son choix. Trop souvent, des lecteurs critiquent une démarche en fonction non pas de l'objectif recherché dans le travail rapporté, mais de leurs propres interrogations sur le phénomène. Sur le plan méthodologique, ces critiques ne sont pas pertinentes.

À titre d'exemple. dans le cadre de la problématique de la fidélité, nous avons cherché à étudier la variabilité spontanée de l'énonciation d'une idée à partir d'une information à communiquer. Nous avons donc conçu une expérience où une information est présentée graphiquement et les sujets sont invités à la formuler verbalement (Gile 1985). Or, il est arrivé que des énoncés produits ne correspondent pas à cette information, quelques participants ne l'ayant pas comprise. Dans ces cas, nous avions exclu ces énoncés de l'analyse, et critiqué sur ce point. Certes, le phénomène de non-compréhension chez ces sujets est intéressant, indique peut-être une faiblesse dans la présentation de l'information aux sujets, et appelle une éventuelle critique sur la réalisation de l'expérience. En revanche, il est étranger au phénomène de variabilité dans l'énonciation d'une même information. La critique sur ce point $n$ 'est donc pas pertinente, de même que l'on ne saurait critiquer la décision du médecin-chercheur d'exclure de son analyse les malades qui n'auraient pas respecté le traitement prescrit faute de le comprendre.

Une fois la ou les questions fondamentales et les objectifs compris, on cherchera a comprendre la réalisation du projet :

La méthode

Pour construire un modele du fonctionnement de l'interprétation, le chercheur peut spéculer en se fondant sur l'introspection. Il peut aussi faire une synthèse des modelles présentés par d'autres chercheurs. Pour mesurer l'effet des cours théoriques dans la formation des traducteurs. il peut choisir de composer un questionnaire à l'intention des étudiants. Pour étudier la différence entre la traduction vers une langue matemelle et la 
traduction vers une langue non maternelle, il peut examiner deux traductions réalisées par la même personne, l'une vers sa langue maternelle, et l'autre vers une langue acquise.

Parfois, la méthode est simple et facile à comprendre. Il arrive aussi qu'elle le soit moins, notamment quand elle repose sur des considérations psycholinguistiques, psychocogniticiennes ou linguistiques d'une grande technicité impliquant des opérations complexes (voir par exemple. Dillinger 1989). Toutefois, même dans un tel cas, il est en général possible, au prix d'un certain effort. de comprendre la logique de la démarche, ce qui en permet une analyse peut-être partielle, mais intelligente.

Les résultats

Une fois la méthode mise en axuvre, elle produit des résultats. Ceux-ci peuvent être assez faciles à saisir: un modèle simple de l'interprétation. une série de réponses à des questions sur l'utilité des cours théoriques dans la formation à la traduction dans un échantillon particulier, une série de "notes" d'évaluation d'une traduction vers la langue maternelle el d'une traduction vers la langue acquise. Parfois, ils sont plus complexes, notamment quand ils se présentent sous la forme technique de statistiques résultant de manipulations compliquées.

- Les conclusions de lauteur

À partir des résultats, le chercheur aboutit à des conclusions. Celles-ci sont en général assè faciles à comprendre.

\section{ÉVALUER LE TEXTE}

Les faits

La recherche scientifique se fonde sur des faits, et se distingue notamment par la manière dont elle cherche systématiquement à les recueillir, à les décrire et à en vérifier la réalité, principe que soulignent les auteurs de textes sur les méthodes de recherche dans toutes les disciplines (voir Boudon 1969: Babbie 1992; Fourastié 1966; FrankfortNachmias et Nachmias 1992: Grawitz 1986; Le Ny 1975: Mahmoudian 1982: McBumey 1990; Reuchlin 1969: Robert 1988).

Une première série de questions que se pose le lecteur critique à propos d'un travail de recherche porte sur les faits, et peut se résumer par l'interrogation suivante: les faits rapportés représentent-ils correctement la réalité ?

Plus concrètement, le lecteur se demandera si l'ensemble des faits rapportés sont suffisamment complets ou représentatifs, s'ils sont pertinents, s'ils sont rapportés correctement, rigoureusement, de manière objective. Notons que l'interrogation porte sur deux types de faits:

- Les phénomènes sur le terrain.

- Les idées. travaux el résultats connus au sein de la communauté des chercheurs qui l'étudient. Nous les appellerons ici faits hibliographiquess, car la plupart sont rapportés dans des textes el se propagent par ce biais.

En traductologie, la première catégorie de faits porte essentiellement sur le comportement professionnel des traducteurs et sur les caractéristiques de leurs traductions, ainsi que sur des comportements cognitifs, langagiers et de communication en dehors de la traduction, mais qui lui sont liés ou qui sont susceptibles d'aider à la décrire ou à l'expliquer: la deuxième porte sur les travaux des traductologues, mais aussi sur ceux des linguistes, des psycholinguistes, des psychologues cogniticiens. des chercheurs en littérature, etc.

Il va sans dire que chaque lecteur ne peut évaluer les faits rapportés qu'en fonction de ses propres connaissances. Il est évidemment d'autant moins bien placé pour le faire 
que le domaine concerné lui est étranger. Dans le domaine traductologique, un grand nombre de lecteurs sont eux-mêmes praticiens, ont une connaissance relativement bonne d'un secteur au moins des faits sur le terrain, et sont en mesure de détecter des imprécisions, erreurs et lacunes dans le texte. C'est ainsi notamment que les interprètes-chercheurs ont décelé de nombreuses erreurs dans les travaux des psychologues et psycholinguistes des années 1960 el 1970 (voir Gile 1993).

En ce qui concerne les «faits bibliographiques", les connaissances qu'ont les traductologues des travaux réalisés dans d'autres disciplines restent souvent faibles, bien que l'on constate ces demières années une nette progression dans ce sens. Des lacunes à cet égard sont regrettables, mais compréhensibles. En revanche, en tant que chercheurs, s'ils ont accès aux revues et ouvrages spécialisés et comprennent les langues concemées, ils ne sauraient ignorer les travaux réalisés par d'autres traductologues sur le sujet qui les intéresse. Or, dans de nombreux textes, aucune référence n'est faite aux idées et résultats pertinents apparaissant dans des textes publiés précédemment, ce qui revient a priver le lecteur d'informations lui permettant de mieux comprendre où se situe le projet par rapport aux connaissances et efforts d'investigation dans la communauté scientifique. S'il n'est pas nécessaire, ni toujours possible d'ailleurs, de citer tous les travaux publiés consacrés à un thème. il convient d'en évoquer les plus marquants, les plus pertinents, ainsi que d'éventuels travaux illustratifs d'une catégorie. En tout état de cause, la méconnaissance des idées et travaux existants est une faute méthodologique : «Le chercheur doit consacrer le maximum d'efforts à formuler des hypothèses significatives, basées sur un inventaire exhaustif des connaissances déjà accumulées en la matière» (Robert 1988: 66). Nous pensons que la lenteur de la progression dans notre domaine au cours des années passées est en grande partie attribuable au fait que de nombreux chercheurs ne prenaient pas la peine de lire systématiquement les idées et résultats pertinents des auteurs qui les avaient précédés, et qu'en conséquence, une grande partie de leurs efforts ont été répétitifs et inefficaces.

Le lecteur se demandera donc si l'auteur fait un rapport suffisamment complet et représentatif des idées et connaissances pertinentes à propos de la problématique qu'il aborde, si les principaux auteurs et les principaux travaux pertinents sont cités. II se demandera aussi si tous les auteurs et travaux cités méritent de l'être, si certains sont cités pour des raisons diplomatiques, voire simplement pour allonger la liste des références, si les idées et les textes sont cités correctement, ou de manière erronée ou fallacieuse.

\section{Le raisonnement}

Une deuxième série de questions sur le fond que se posera le lecteur critique relève du raisonnement, où l'analyse est moins tributaire de ses connaissances thématiques. La part relative des connaissances thématiques et de la logique dans la lecture critique varie. Il existe toutefois un consensus dans les disciplines scientifiques sur une logique de recherche fondamentale, qui est cartésienne et explicite, et sur la nécessité de la rigueur dans l'étude et l'exposé des faits, mais aussi dans le raisonnement.

Les critiques de fond sur le raisonnement peuvent porter sur l'ensemble des choix du chercheur, mais au regard des conventions qui ont cours en matière de recherche scientifique, elles n'ont pas toutes le même poids. Ainsi, la problématique choisie peut paraître marginale ou inintéressante à un lecteur, mais la liberté du chercheur de choisir son sujet ne semble pas être fondamentalement contestée. Notons que sur le plan pratique, pour l'obtention de bourses de recherche ou de subventions ou pour le recrutement dans des institutions de recherche, cette liberté apparaît quelque peu théorique, et dans la recherche industrielle, le choix de la problématique est primordial, mais au regard de la démarche de recherche proprement dite, elle semble consensuellement admise. 
Le choix des objectifs précis du travail concerné est-il justifié?

Ce choix, lui, peut prêter le flanc à une critique plus fondamentale. en ce sens qu'on peut juger les objectifs précis du travail comme plus ou moins pertinents ou plus ou moins prioritaires au regard de la problématique fondamentale. Ainsi, concernant les difficultés de la transmission informationnelle en interprétation, un chercheur a eu l'idée d'étudier les vibrations des cordes vocales des apprenants de l'anglais langue étrangère lisant des textes dans cette langue. Il paraît difficile d'établir un lien entre cette exploration et la problématique dont elle est supposée relever. En revanche, dans le cadre de la même recherche, on trouvera appropriée l'étude des dégradations de la prestation face à des obstacles particuliers : bruit ambiant, discours rapide, débit de l'orateur.

Toutefois, c'est bien en aval de ces choix que se situent les articulations les plus critiques d'un travail de recherche.

Les moyens choisis par le chercheur pour réaliser ses objectifs sont-ils appropriés?

C'est là que se reflète en général l'essentiel de l'effort méthodologique et pratique du chercheur dans son projet. Il s'agit de bien choisir une ou plusieurs méthodes et de bien les mettre en auvre, ce qui pose de nombreux problèmes conceptuels et pratiques. Parmi les écueils sur lesquels achoppent le plus fréquemment les chercheurs, citons:

L'échantillonnage: échantillons trop petits ou non représentatifs. Les problèmes d'échantillonnage se posent dans la plupar des disciplines expérimentales. En traductologie, les problèmes les plus fréquents se rapportent à l'utilisation d'étudiants, voire de «bilingues" non familiarisés avec la traduction et l'interprétation, dans des expériences supposées fournir des données sur la traduction ou l'interprétation.

Les mesures: problèmes de validité, de fiabilité, de précision. L'évaluation de la qualité du travail donnée par un interprète est-elle une mesure valable de l'impression sur la qualité du travail telle qu'elle se cristallise peut-être chez un délégué? L'évaluation donnée face à face et explicitement à un traducteur concernant son propre travail mesuret-elle valablement ce que ressent véritablement le client? Les méthodes choisies pour mesurer la qualité du travail vers la langue maternelle et vers la langue non maternelle permettent-elles une bonne comparaison des deux? Peut-on considérer qu une mesure faite à un moment donné correspondra à une mesure faite à un autre moment sur le même phénomène? Les réponses à une interview sont-elles suffisamment précises? Les mesures du débit informationnel d'un discours sont-elles appropriées? (Voir, par exemple, les critiques de Catherine Stenzl (198.3) sur les mesures de qualité de l'interprétation réalisées par Henri Barik et David Gerver.)

Les environnements expérimentaux ne présentant pas une similitude suffisante avec l'environnement réel : de même qu'en psychologie, en traductologie, certains contestent la validité des environnements expérimentaux pour l'étude des phénomènes sur le terrain. On peut aussi s'interroger sur la mesure dans laquelle une situation scolaire de traduction ou d'interprétation peut éclairer le chercheur sur un phénomène se produisant sur le terrain. Sans aller jusqu'à ces objections de fond. le lecteur peut juger de l'efficacité des efforts du chercheur pour rendre l'environnement expérimental aussi proche que possible des conditions authentiques de traduction ou d'interprétation.

Les tâchess expérime'ntale's ne représentant pas valablement les tâches quétudie le chercheur (exemple d'interprétation de mots isolés en laboratoire, d'interprétation dans des combinaisons linguistiques inhabituelles dans le cadre d'études sur l'interprétation (voir Anderson 1979), d'interprétation de «discours» artificiels ne présentant pas a priori les mêmes caractéristiques que les discours interprétés en conférence (voir Gile 1990)).

L'emploi incorrect des techniques statistiques: à l'heure actuelle. ce problème $n$ 'affecte pas fortement les traductologues, car ceux-ci ne font pas souvent appel aux statistiques inférentielles. Il est toulefois endémique chez les médecins (voir fore et 
Altman 1982), ainsi que chez les biologistes et psychologues, et mérite donc d'être souligné.

Les conclusions de l'auteur sont-elles justifiées?

Plus encore que les autres parties du travail de recherche. les conclusions sont une question de logique et de bon sens. Le lecteur critique se demandera si les conclusions tirées par l'auteur découlent vraiment des résultats obtenus, si ces résultats apportent vraiment la démonstration du bien-fondé de son hypothèse, s'ils ne peuvent pas être expliqués autrement, si l'auteur n'a pas généralisé au-delà de ce qui est raisonnable compte tenu des méthodes et de l'échantillon utilisés.

Par exemple, compte tenu de la petite taille d'un échantillon et de sa sélection dans un environnement géographique et linguistique très localisé, peut-on vraiment tirer des conclusions générales sur l'ensemble de la population des traducteurs? Le fait que différents interprètes notent différemment en interprétation consécutive des concepts apparaissant dans le discours original prouve-t-il vraiment qu'il y a déverbalisation entre réception et reformulation? La variabilité n'est-elle pas possible même si les mots en langue d'arrivée sont évoqués directement par la langue de départ? Si tel traducteur semble effectivement avoir mieux traduit tel texte vers une langue acquise que vers sa langue matemelle, peuton en conclure que les traducteurs dans leur ensemble ne traduisent pas mieux vers leur langue matemelle que vers une langue acquise, ou peut-il s'agir d'une exception ou d'un cas particulier qui s'explique autrement?

Dans la traductologie, la faiblesse la plus fréquente à cet égard semble être une extrapolation excessive dans le sens de l'hypothèse ou de la théorie privilégiée du chercheur, ce qui est peut-être caractéristique d'un manque de maturité méthodologique de la discipline. À ce sujet, on étudiera avec profit les textes de recherche dans des disciplines plus anciennes, où les conclusions sont marquées par une grande prudence.

\section{La présentation}

Étant donné la nature «sociale», "publique» et «cumulative» de la science (Moravcsik 1980: 40), donc son aspect communicationnel, la valeur d'un travail de recherche tient non seulement aux faits et aux raisonnements qui le composent, mais aussi à sa présentation.

À cet égard, les deux maîtres-mots à évoquer sont la clarté et l'explicitation. La clarté est liée au style de l'écriture. mais aussi à la mise en pages et aux tableaux et graphiques, comme dans tout autre texte documentaire. L'exigence d'explicitation, elle, est particulièrement forte dans les textes scientifiques, précisément pour en permettre la lecture critique et la réplication: «Une démarche est dite scientifique si elle autorise d'autres chercheurs à reproduire les observations, à éprouver à leur tour les hypothèses et a les réfuter le cas échéant" (Robert 1988: 6; voir aussi, Babbie 1992:19; Reuchlin 1969 ; Spiegel 1956:6).

Au-delà de ces deux paramètres fondamentaux, on pourra évoquer tous les autres facteurs de lisibilité, qui peuvent rendre le texte plus facile, voire plus agréable à lire : fluidité et rythme, choix lexical, mise en pages, choix et formes des illustrations, typographie.

Il existe en outre dans la recherche certaines exigences formelles. qui portent sur la morphologie générale des textes, sur le style, la présentation des citations, la bibliographie. Ces exigences varient quelque peu selon la discipline, le type de texte, voire le pays ou l'université concemée (surtout s'agissant de thèses). Dans certains cas, le respect rigoureux des règles de présentation peut cacher une grande faiblesse sur le fond. À ce propos, on ne peut que savourer l'excellent Cantatrix Sopranica $L$. de Georges Perec (199I), où l'auteur écrit des pastiches d'articles scientifiques en respectant scrupuleusement les conventions de présentation, mais dont le contenu est fantaisiste. 
L'évaluation de la présentation est minutieuse dans deux cas : celui de l'autocritique, notamment avant soumission du texte à un jury de thèse ou à une revue. et celui où le lecteur est précisément membre d'un jury ou d'un comité de lecture. Dans la plupart des autres circonstances, le lecteur ne réagit consciemment qu'à des caractéristiques de présentation qui le frappent, quand des passages ne sont pas clairs, quand ils ne sont pas suffisamment explicites, quand la bibliographie ne lui donne pas tous les renseignements recherchés, ou au contraire quand il trouve le texte particulièrement clair et agréable à lire.

\section{SYNTHESE : L.A VAL.E.UR (;I.OBAI.F DU TEXTE}

L'analyse étant faite sur les différents aspects du texte, se pose la question de la synthèse, à savoir la valeur globale du texte. Celle-ci dépend non seulement des jugements personnels de chaque évaluateur sur ses différentes composantes, mais aussi de l'optique dans layuelle le texte a été lu. catégories.

Par commodité, il peut être intéressant de grouper les variables en différentes

- L'innoration: l'innovation justifiée est synonyme de progression, et se place donc très haut dans l'échelle des valeurs scientifiques (du moins théoriquement, car dans la pratique, comme le souligne Thomas Kuhn, si elle bouscule les idées reçues, elle peut être très mal reçue au sein de la communauté scientifique). Le caractère original des travaux, qui fait partie des exigences universitaires pour des thèses de doctorat et pour des recrutements, des bourses de recherche, voire des prix, n'est pas autre chose que cet aspect innovateur. Le lecteur pourra se demander dans quelle mesure le texte innove par les faits (faits nouvellement découverts sur un phénomène. résultats d'une procédure expérimentale), par les idées (nouvelle analyse. nouvelles hypothèses), par les méthodes (création ou adaptation d'outils intellectuels ou matériels). L'importance de l'innovation apportée, quant à elle, $n$ 'est pas toujours facile à évaluer. Si la mise au point d'un vaccion contre une maladie grave est reconnue comme très importante d'emblée. de nouvelles hypothèses ou théories peuvent mettre longtemps avant de faire leur chemin dans la communauté scientifique, et l'imponance de la découverte d'un nouveau fait peut n'apparaître qu’à la lumière d’autres découvertes, bien plus tard.

- Le respect des normes scientifiques: il se mesure en rigueur. en précision, en objectivité dans le traitement des faits, des hypothèses. des raisonnements. Il s'évalue aussi au regard du respect des normes de présentation mentionnées plus haut. Un travail peut en effet être innovateur mais insuffisamment rigoureux ou précis. Inversement. il peut être conforme aux normes scientifiques, mais n'apponer que très peu d'éléments nouveaux.

- La qualité communicationnelle du texte' celle-ci est fonction de la clané du raisonnement suivi et des faits exposés, ainsi que des autres paramètres de présentation.

- Les qualités morale's de l'aute'ur: on appréciera son séricux à travers son objectivité et sa rigueur, notamment dans le soin qu'il met à hien rechercher les faits pertinents. y compris les faits bibliographiques. el à les vérifier. On appréciera aussi son intégrité telle qu'elle peut apparaitre (ou ne pas apparaitre) dans l'attribution explicite de la paternité des idées à ceux qui le méritent et dans la référence à des auteurs et leurs travaux. même si leurs idées ne concordent pas avec less siennes.

Au-delà de la variabilité personnelle des appréciations, la pondération de chacun de ces paramètres dépend du type de texte concerné et de sa destination. Par exemple, pour les membres d'un jury de thèse, le respect des normes scientifiques est de première importance, mais on ne s'attend pas de la part du jeune chercheur à de grandes innovations. En revanche. s'agissant de l'attribution d'un prix. l'innovation prime. Dans les revues scientifiques de haut niveau. tous les critères de fond et de présentation ont une grande 
importance. L'existence d'un groupe de revues de très haut niveau où la sélection des articles est sévère, à côté d'un grand nombre de revues «mineures", semble être une caractéristique des disciplines solidement établies dans la communauté scientifique, et offre aux jeunes chercheurs des exemples de publications à suivre. La traductologie ne nous semble pas encore avoir atteint ce stade de maturité, et la qualité des articles et communications publiés reste souvent trop inégale.

\section{CONCLUSION}

On ne saurait trop rappeler la nature auto-correctrice de la recherche scientifique. On ne saurait trop rappeler qu'en traductologie, en l'absence d'un ensemble de programmes de formation à la recherche, cette auto-correction passe, bien plus que dans d'autres disciplines plus mûres, par la lecture critique. Nous avons cherché dans cet article à proposer une grille pour une telle lecture, et espérons qu'elle aidera les jeunes chercheurs à mieux évaluer les textes qu'ils liront et à améliorer leurs propres textes en fonction des mêmes critères. Nous espérons aussi avoir montré que la lecture critique peut être fructueuse même quand les connaissances techniques du lecteur sont modestes. Un "candide» raisonneur peut beaucoup apprendre en lisant de manière critique les textes d'un chercheur chevronné. Il peut aussi y détecter d'éventuelles lacunes; personne, pas même les scientifiques les plus connus, n'est à l'abri d'une erreur (McBurney 1983: 16). À force de travailler dans le cadre d'un même paradigme, les chercheurs chevronnés peuvent d'ailleurs se retrouver dans des ornières qui les rendent aveugles à des faits et idées qu'un observateur naïf verra aisément et qui, comme il est expliqué plus haut, lui indiqueront une direction à suivre. Rappelons, pour conclure, que la science est bâtie dans une très grande mesure non pas sur des techniques, mais sur le bon sens. Comme le dit Peter Medawar, prix Nobel en médecine, the scientific method, as it is sometimes called, is a potentiation of common sense (1979: 93). Et le bon sens n'est-il pas la chose au monde la mieux partagée?

\section{REFERENCES}

ANDERSON, Linda (1979): Simultaneous Interpretation: Contextual and Translation Aspects. M.A. Thesis. Concordia University, Montreal.

BABBIE, Earl (1992): The Practice of Social Research. Belmont, California, Wadsworth Publishing Company. BOUDON. Raymond (1969): Les méshodes en sociologie. Paris, PUF.

CHAUVIN, Rémy (1981): Des savants pour quoi faire? Paris, Payol.

DANCETTE, Jeanne (199()): Etude reflexive et experimentale du processus de comprehension dans / activite de radurtion, thèse de doctorat, Université de Montréal.

DILLINGER, Mike (1989): Component Processes of Simultaneous Interpreting. PhD Thesis, Department of Educational Psychology. McGill University. Montreal.

FOURASTIF́. Jean (1966): Les conditions de l'espris scientifique. Paris, Gallimard.

FRANKFORT-NACHMIAS, (hava et David NACHMIAS (1992): Research Methods in the Social Sciences. London, Melhoume, Auckland, Edward Amold.

GILE, Daniel (1985): “De l'idé a l'enonce : une expérience et son exploitation pédagogique dans la formation des traducteurs", Meta, 30-2, pp. 139-147.

GILE, Daniel (1990): "Observational Studies and Experimental Studies in the Investigation of Interpretation» Communication faite a la Scuola Superiore di Lingue Modeme per Interpreti e Traduttori, Universita degli Studi di Trieste, le 19 mars 1990.

GILE. Daniel (199.3): «Opening up in Interpretation Studies», in Mary Snell-Homby, Franz Pochhacker et Klaus Kaindl (Eds.). Translation Studies. An Interdiscipline, Amsterdam/Philadelphia. John Benjamins. $\mathrm{XI}+4.39 \mathrm{p}$

GRAN, Laura and Christopher TAYLOR (Eds.) (1990): Aspects of Applied and Experimental Research on Conference Interpretation, Udine, Campanotto Editore.

GRAWIT7. Madeleine (1986): Méthodes des sciences sociales, Paris, Dalloz.

HERMANS. Theo (Fd.) (1985): The Manipulation of Literature. Studies in Literary Translation, London, Croom Helm. 
KRINGS, H.-P. (1988) : ${ }^{\text {B }}$ lick in die 'Black Box' - Eine Fallstudie zum Übersetzungsprozess bei Berufsübersetzern". R. Amtz (Ed.). Texilinguistik und Fachsprache. Akten des Internationalen ühersetzungswissehschaftlichen AlLA-Symposiums Hildesheim. 13-16, April 1987, Studien zu Sprache und Technik. Band 1. Hildesheim, Olms, pp. 393-411.

LE NY, François (1975): “Sémantique et Psychologie», Langages, n 40. decembre 1975.

LORSCHER, Wolfgang (1991): Translation Performance, Translation Process, and Translation Strategies A Psycholinguistic Investigation. Tübingen. Günter Nart.

MAHMOUDIAN, Morteraa (1982): La linguissique, Paris, Seghers.

McBURNEY. Donald H. (1990): Experimental Psychology. Belmont, California, Wadsworth Publishing Company.

MEDAWAK, Peter B. (1979) : Adsice fo a Young Scientist, London and Sydney, Pan Books.

MORAVCSIK, Michael (1980): How to Grow' Srience, New York, University Books.

MOSER-MERCER, Barhara (1991): «Research Committee - Paradigms Gained or the An of Productive Disagreement», Bulletin de l'Al/C, 19-2. pp. 11-15.

NAGEL, Emst (1967): "The Nature and Aim of Science», Sydney Morgenbesser (Ed.). Philosophy of Science Today, New York and London. Basic Books Inc.. pp. 3-13.

PEREC. Georges ( 1991$)$ : Cantatrix Soprancia l... Paris. Seuil.

REUCHLIN. Maurice (1969): Les méthodes en psychologie, Paris, PUF

ROBERT. Michèle (1988) : Recherche scientifique en psycholegie. Saint-Hyacinthe (Quebec), Edisem et Paris, Maloine.

SEGUINOT, Candace (1989) : The Translation Process, School of Translation, York University, H.G. Publications.

SPIEGEL. Sidney (1956): Nonparametric Statistics for the Behusioral Sciences. New York. Toronto and London. McGraw-Hill Book Company.

STENZI. Catherine (1983): Simulituneous Interpretation - Groundwork Towards a Comprehensive Model. M.A. Thesis, Birkbeck College, University of London.

TIRKKONEN-CONDIT, Sonja (Ed.) (1991): Empirical Research in Translation and Intercultural Studies. Selected papers of the TRANSIF Seminar, Savonlinna 1988. Tübingen. Gunter Narr

TIRKKONEN-CONDIT, Sonja and John LAFFI.ING (Eds.) (1993): Re'cent Trends in Empirical Transiarion Research. Studies in Language'. $n^{\circ} 28$, University of Joensuu.

TOURY, Gideon (1985): "A Rationale for Descriptive Translation Studies". Theo Hermans (Ed.). The" Manipulation of Literature. Studie's in Literary Translatıon. London and Sydney, Croom Helm, pp. 16-41. 